\title{
The Fundamental Theorem of Algebra
}

\author{
Adrian Throop
}

Math 4495

Dr. Derado

Fall 2005 


\section{The Fundamental Theorem of Algebra}

Of the options, the Fundamental Theorem of Algebra was chosen to be investigated. A majority of the project has been dedicated to a proof of the theorem, and the remainder is dedicated to discussion about the theorem and it's origin and relevance to the high school math environment.

As is typical in discussion of mathematical theories and theorems, the theorem is stated. The Fundamental Theorem of Algebra states that any complex polynomial of degree $n$ has exactly $n$ roots. Another simple way to state the theorem is that any complex polynomial can be factored into $n$ terms. It will be discussed later that neither of these forms is quite how the theorem was stated in it's original proof by Carl Friedrich Gauss. In fact, the theorem can be stated in a number of ways while still retaining the same meaning. Before delving into particulars on this, let's talk about the reasons behind the necessity of discussing (and furthermore proving) the theorem. We will try to take an approach to answer those age-old type questions we hear from high school students.

\section{"What in the world does the theorem say"}

Telling a high school student that we can take any complex polynomial and find all $n$ roots of it no matter what polynomial we are given may be a bit like telling a small child that gravity is a big reason why it hasn't yet figured out how to walk. The first clarification then may be to explain what is meant by the word "root." When we say an equation has a root, we mean that it has a value that evaluates it to zero. For instance, $f(x)=7 x-14$ will evaluate to zero when $x$ is 2 . An early level algebra student should be able to grasp this.

In order for a more complete understanding, the root must be taken, literally, to a higher degree. When a student is told that $f(x)=x^{4}+2 x^{3}-10 x^{2}+7 x-5$ will have 4 roots by virtue of the theorem, it becomes almost immediately abstract to them. However thanks to technological innovations such as graphing calculators, equations of the 4th degree (or higher) can be shown to have 4 (or more) roots by showing that the equation crosses the x-axis in 4 places. Of course, when an equation with real coefficients has any complex roots, the graphing calculator will only take the student so far. Consider $f(x)=x^{3}-1$; this has only one $\mathrm{x}$-intercept, but the student is told that it will have 3 roots. Here is a big catch with the fundamental theorem, remember it was stated for all complex polynomials.

This means that the student will be told that the fundamental theorem applied to real polynomial equations will hold in that there will still be $n$ roots; its just that the student won't be allowed to see all these roots on his or her calculator. This may anger the student. Furthering their anger will be that their calculators can't display complex polynomials. Rather than having student bodies run riot over the fundamental theorem, the idea is to reconcile the idea of real polynomials with the statement of the fundamental theorem.

So although a student will not be able to see the roots of an equation on their 


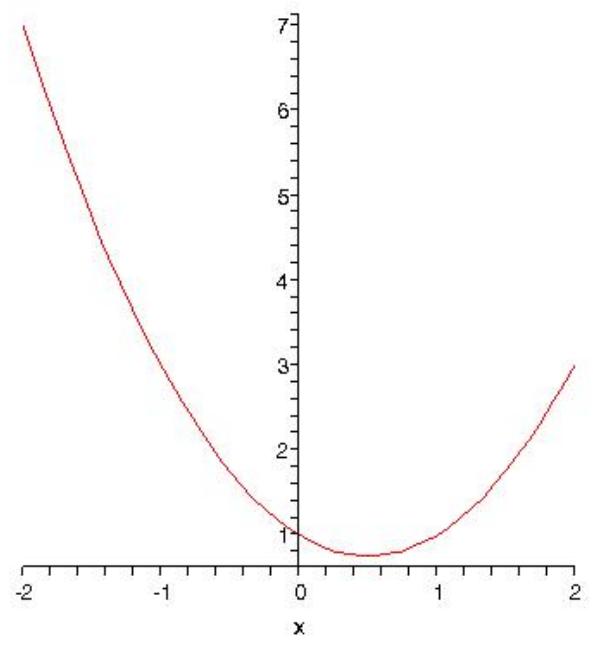

Figure 1: A function with no real roots

calculator, they are taught that with just a little algebra they can at least get to see the roots on paper. Let's use $f(x)=x^{2}-x+1$, and a student is asked to find the roots of the this equation. The student may begin by trying, again, to use the calculator. This will immediately give the student the speed bump just discussed.

Hopefully the student is paying close attention and will not say, "The equation has no roots!" Instead he or she is expected to first try to factor the polynomial into terms of the form $(x-a)$. Finding this cannot be done, the student will not be discouraged, moving onto the old-reliable quadratic formula. With sufficient execution of the formula, along with elementary knowledge of complex numbers, the student should finally produce the two roots of the equation,

$$
\frac{1}{2}+\frac{i \sqrt{3}}{2} \quad \frac{1}{2}-\frac{i \sqrt{3}}{2}
$$

A few more examples such as this may begin to help the student to understand that a root is any number which evaluates an equation to zero. A fallacy may arise here, because all that has been done is a new name given to something that the student has already seen enough of. Which leads to the next stereotypical high school student response to things with the seemingly ambiguous nature of the fundamental theorem.

\section{"Ok. So what?"}

At this point, the student's understanding of the fundamental theorem is such that the fundamental theorem states that for any polynomial equation we will be able to find answers using our calculators or quadratic formula, etc. "Yeah. So what?" may be exactly the student's response to this. Every equation they have been asked to solve with the quadratic equation has always been solvable. The theorem will not be impressive to 
them at all! In fact, they may just assume their time is being completely wasted.

For now we must leave the student's frustrations only to say that the origins of the fundamental theorem actually come about from very similar notions. Approaches similar to that of the quadratic formula date back further than 2000 years. The earliest algebraic representations of the formula are credited to Brahmagupta in India in the 7th century. By the 12th century, another Indian mathematician, Bhāskara, had gotten so formal as to state "The square of a positive, as also of a negative number, is positive; that the square root of a positive number is twofold, positive and negative. There is no square root of a negative number, for it is not a square." (1)

At this point in history, the inference is made that perhaps mathematicians still had a notion that certain equations had roots while others did not. However by the time of Euler, the ideas revolving around complex numbers had been thoroughly (though not completely) developed. So, just like a high school student using the quadratic formula ad nauseum might conclude, mathematicians began to accept that all polynomials would have $n$ roots. And like high school students might, some mathematicians thought this would be a silly thing to worry about proving. Still, there is no quadratic equation for polynomials of higher than degree 5 , so how is it that we can be assured that the roots are there?

To answer this question we of course need the Fundamental Theorem of Algebra. However, the need for the theorem hardly produced the theorem out of thin air. The first formal attempt to prove the theorem was by Albert Girard in a 1629 publication entitled L'invention en algèbre (5). In it he used inductive reasoning to say that any equation of degree $n$ will have $n$ roots, and he even mentioned that negative numbers may have imaginary solutions, but he was not yet able to quantify it other than to say that the solutions were there somewhere (1).

Throughout the 18th century, further attempts were made to fully understand and prove the theorem. Jean le Rond d'Almbert made the first published attempt in 1746, but his proof was flawed, and among other things assumed that the theorem was true in order to prove it. Despite his shortcomings, the theorem still partly bears his name in France. Three other big names of the 18th century, Euler, Laplace, and Lagrange, each had their own attempts at the theorem as well, but each was not able to produce a complete proof (5).

In 1799, Gauss produced the first proof of the theorem, although as we will mention in a moment it was not the theorem in it's current form. His reasoning was later found to have gaps, but still this is referred to as the first complete proof of the theorem. Gauss would later publish three further proofs of the theorem, however before these would come Argand's proof (4).

Seven years after Gauss' first publication, Jean-Robert Argand, a bookseller, published another proof of the theorem, but Argand avoided the gaps that Gauss had. Argand's association with the theorem proceeded to become very widespread in part from his earlier accomplishments with complex numbers, including the defining of the argument of a complex number. The term and likewise the notation (Arg) of a complex number are attributed to him; he is remembered as being one of the first mathemati- 


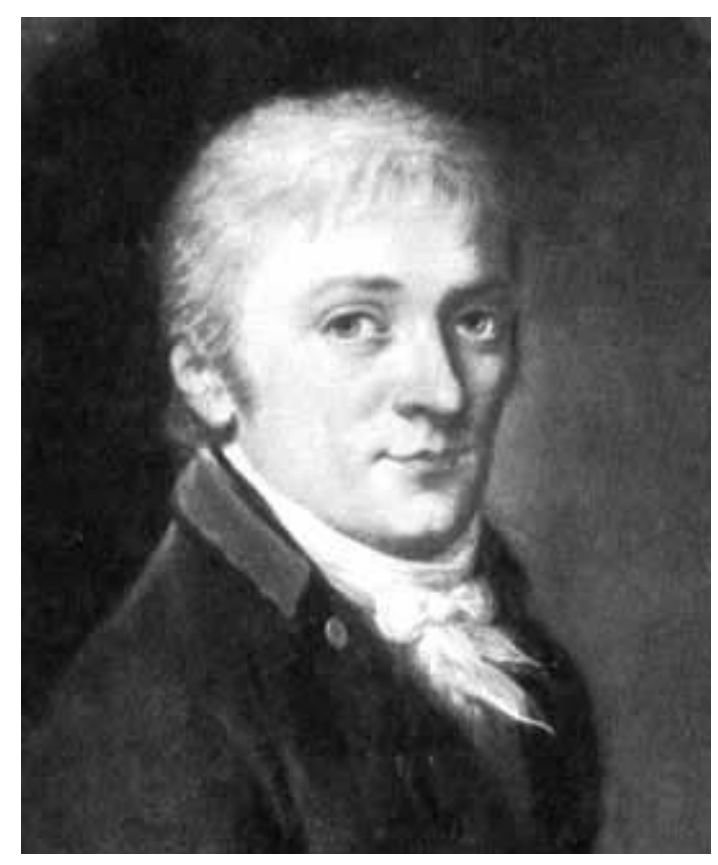

Figure 2: Gauss, c.1803

cians to include absolute magnitude with the visualization of a complex number. Even though Argand is commonly credited with establishing the complex argument, he may have actually gotten the idea from Caspar Wessel. It has been found that Wessel had the idea for the complex argument a few years ahead of Argand, however Wessel was born in Norway, and not much was made of Scandinavian mathematicians. Combining this with Argand's French heritage (a more highly respected mathematical locale at the time), credit has always leaned in Argand's favor in terms of the visualization of complex numbers (1).

Still, by the early 19th century the theorem had been proven. However Gauss would not be outdone. As mentioned earlier, he moved on to prove the theorem in three different ways. His 4th proof published in 1849 turned out to be his crowning achievement with the fundamental theorem. It was the first proof that allowed the polynomials to have complex numbers. Like high school students, Gauss and others could only deal with polynomials of real coefficients at first (4).

Without investigating proof of the theorem, it is hard to quantify what exactly makes the theorem so difficult. Just from a few of the historical facts surrounding the theorem we can uncover it's difficulty. The theorem had been circulating major mathematical circles in the 18th century, and Euler couldn't even prove it. Gauss' first try had it's own gaps, and it wasn't until his fourth proof that a more or less complete proof had been produced.

This also begs another question when you consider the theorem's analog, the Fundamental Theorem of Arithmetic. This theorem says that any number can be written uniquely as a product of it's prime factors. It is commonly observed that this is a sim- 
ilar idea to stating a polynomial can be written as a product of it's factors, again the Fundamental Theorem of Algebra. If these two are so similar in form and nature, why is it that the Fundamental Theorem of Arithmetic was proved thousands, rather than hundreds, of years ago by Euclid (4)? And if the Fundamental Theorem of Arithmetic is that much easier to handle (but no less important in finite mathematics and number theory), why do we see the Fundamental Theorem of Algebra appear in the high school curriculum so much more than it's simpler counterpart? It is beyond the scope of this paper to answer questions such as these, yet it is important to see that the modern day relevance and importance of the Fundamental Theorem of Algebra is more than skin deep.

Next in discussion of the theorem is the proof. What is presented is given to be a version of Argand's proof, and was chosen so for both for it's conciseness and visual aspects. The proof's outline was taken directly from a publication by Heinrich Dörrie entitled "100 Great Problems of Elementary Mathematics." 


\section{Proof}

The proof presented is the author's best explanation of Cauchy's modification of Argand's proof of the theorem. Let's begin by stating again the theorem.

Fundamental Theorem of Algebra 1 Any equation of the form

$$
z^{n}+C_{1} z^{n-1}+C_{2} z^{n-2}+\cdots+C_{n}=0
$$

has at least one root.

The Fundamental Theorem is presented in two parts. First, it will be shown that every polynomial equation has at least one root. Once this is shown, it can be used to establish every polynomial equation of degree $n$ having $n$ roots. To begin, we need to discuss the approach to the proof. The form of the equation discussed is shown equal to zero, and herein lies our starting point. It will be shown that there will always be a specific value for $z$, say $z_{0}$, that will evaluate the given polynomial to zero. With the help of complex visualizations of the polynomial, a two case scenario will be shown. The main aspects of the proof is to show the impossibility of one of these cases. This is done indirectly through contradiction. The contradiction shows that for $z_{0}$, the polynomial at $z_{0}$ must evaluate to zero and nothing else.

Begin with a function, $f(z)$. Let the function be the equation we are trying to prove.

$$
f(z)=z^{n}+C_{1} z^{n-1}+C_{2} z^{n-2}+\cdots+C_{n}=0
$$

This is a form of the equation given by the theorem with which we will use. Before doing so, it will be good to discuss the function with some depth.

The function established is a function of $z$, a complex variable (which can also be referred to as an imaginary number or variable). Likewise, the coefficients, $C_{i}$, are complex constants. It may not yet be clear why dealing with the complex numbers is preferred, however when further finding any equation has exactly $n$ roots it's use will become much more apparent. For now, it is good to note that any real number can be written as a complex number by simply letting the complex part be zero (in practice we see the real number, 5 , as it's complex counterpart, $5+i \cdot 0)$. So, we observe that $f(z)$ is written in a complex form, but may also contain components which are real numbers.

Now we introduce a value for $f(z)$. We call it $\omega$, and thus,

$$
f(z)=z^{n}+C_{1} z^{n-1}+C_{2} z^{n-2}+\cdots+C_{n}=\omega
$$

Why do we make this change? It seems like a step back from trying to prove that the function is equal to zero, but the introduction of $\omega$ is in actuality more like aiming a weapon rather than firing wildly. Simply consider $\omega$ to be all the values of the function for different values of $z$. Now we show that there is at least one particular $z_{0}$ that 


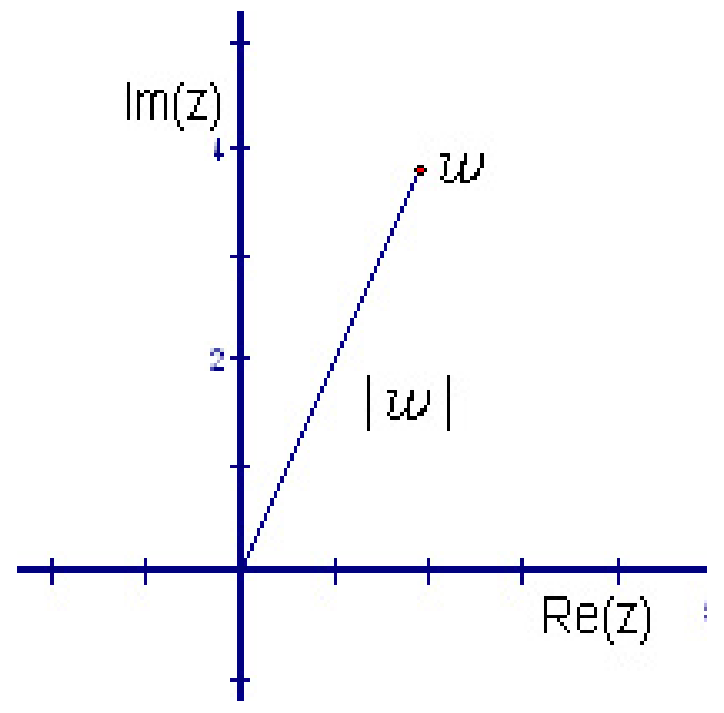

Figure 3: $f(z)=\omega$ for some $f(z)$

evaluates the function, and $\omega$, to zero. Further, we show this can be achieved for all polynomial assignments of $f(z)$.

Next, let's use some knowledge about complex polynomial functions. When these functions evaluate, they always have a complex number as an output. That is, given any value of $z, f(z)$ will output some number of the form $a+i \cdot b$. Using this form we can visualize values of $f(z)$ on a rectangular plane by way of a real axis and an imaginary axis. We now take a look at where our values for $\omega$ may appear.

Now it is good to discuss the smallest value of $|\omega|$ for any $f(z)$. How do we know we have a smallest value? First recall that $|\omega|$ can take the value of zero at the origin, and that it cannot be less than zero, as an absolute distance is always positive. We then say that the set of all absolute magnitudes is bounded on the left by zero, and we write $|\omega| \in[0, \infty)$. We denote the minimum of these $\mu$.

Now we wish to know what $\mu$ is, and in turn force it to be zero. We certainly know that $\mu$ can be zero, but in that sense we must also consider $\mu$ not being zero. So, we have two cases: $\mu=0$ and $\mu>0$ (remember, $\mu$ is a particular $|\omega|$, which must be positive).

If we have $\mu=0$, the proof is complete. In the second case we consider $\mu$ to be greater than zero, and we show that this case is false for all $z_{0}$. To do this, we begin by assuming that $\mu>0$ is true. The value $z_{0}$ will denote the value of $z$ that results in the smallest absolute magnitude.

$$
\begin{gathered}
f\left(z_{0}\right)=\omega_{0} \\
\left|f\left(z_{0}\right)\right|=\left|\omega_{0}\right|=\mu
\end{gathered}
$$

Now it is good to talk about $\omega_{0}$. As discussed earlier it is simply some value on the complex plane. But now, since we have chosen it to be the smallest, it is subsequently the value closest to the origin. Even though $\omega_{0}$ is the smallest absolute magnitude, there will be values with magnitudes close to $\omega_{0}$. We know this for certain because polynomial 
equations are always continuous. Remember that the form of $f(z)$ is a sum of powers of $z$, and this is such that we can evaluate $f(z)$ for any choice $z$. With our value $z_{0}$, we can pick other values of $z$ that are in the neighborhood of $z_{0}$; that is they are close to $z_{0}$. So, for any $f\left(z_{0}\right)=\omega_{0}$ there will be nearby values $f(z)=\omega$. This also means $z_{0}$ 's neighbors will have absolute magnitudes close to that of $\left|\omega_{0}\right|$, thus other small values of $|\omega|$.

Examining of the neighbors of $z_{0}$ is one of the more vital steps to the proof, so it is well served to expound upon it. A point of clarity is that we are actually examining two neighborhoods of $z_{0}$. The first is the value of $z_{0}$ itself, and the second is that of absolute magnitude of $f(z)=\omega$ evaluated at $f\left(z_{0}\right)$. In other words we have to look at the neighborhoods of both the domain, $z$, and range, $\omega$, of the polynomial function.

The difficulty in trying to examine both of these neighborhoods is that both $z$ and $\omega$ are complex numbers; visualization of both values at once is a tricky task. Instead, splitting them into two, lets begin with values in the domain, $z$. When considering $z$ the first intuition is to limit ourselves to complex numbers which solve the equation $f(z)=0$. This is not the approach to take; remember, we have taken a step back to look at the function in a more open-ended sense when $f(z)=\omega$. Now we can see that we are dealing with all of the complex numbers, as $f(z)$ is simply real powers of complex numbers multiplied by various complex coefficients. Is this something we really want to happen? We are attempting to force $f(z)$ to be zero in a very particular sense for some $z_{0}$, so how does it serve to step back into a case where we have infinite solutions, a virtual opposite of our goal? For the time being, it is best to just allow this frustration to exist and hope that something happening in the range (remember, $\omega$ ) will handle this complication.

Before getting anxious about $\omega$ we must complete the consideration of $z_{0}$ 's neighborhood. What do we mean by neighborhood? We want to consider values of $z$ that are close-by $z_{0}$, values that are very much like $z_{0}$; we do this in order to paint $z_{0}$ into a corner where we can tell exactly what it is doing. So to examine the neighborhood of $z_{0}$ we will create a circle with a radius of our choosing with a center at $z_{0}$. Thereby we create $z_{0}$ 's neighborhood, as it is all values of $z$ which lie inside or on the circle. The following is a graph of the complex plane in which we have designated some value to be $z_{0}$ and it has it's corresponding circle which we just discussed.

This picture needs a few clarifications. What we have is a graph of the entire complex number field, or in other words every complex number is on this graph. It is vital to note that this is not the graph on which $f(z)$ lies. This is simply a visualization of the domain; remember, in functions of real numbers we can simply use a number line to consider the domain. We do not have the convenience of a single number line when using complex numbers, as each number is composed of it's real and complex parts, it becomes impossible to have a strict ordering like with the real numbers. So, in the graph above, every point inside the circle is in the neighborhood of $z_{0}$. Is this consistent with what we mentioned earlier? We now have a group of values near $z_{0}$, so it remains to be said that all of these numbers are valid choices for evaluating $f(z)$.

We also need to consider the neighborhoods of $f(z)$ and $\omega$. Since we have defined 


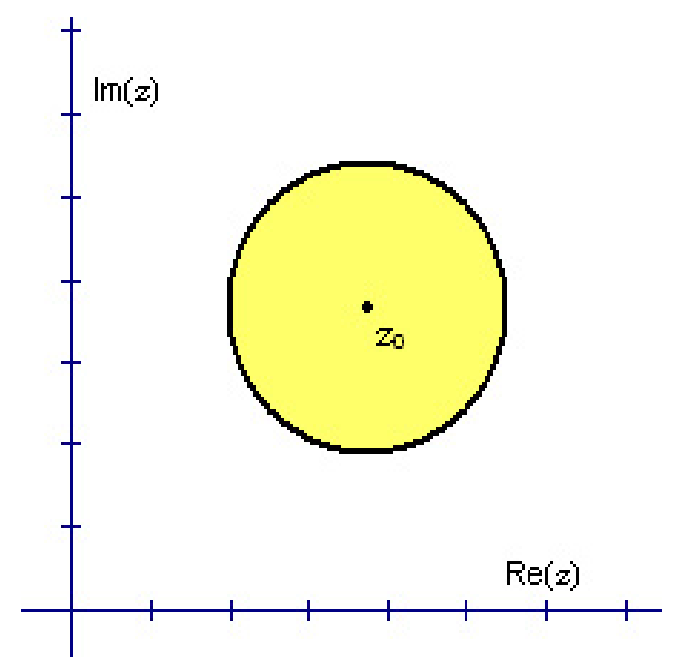

Figure 4: Visualization of $z_{0}$ in domain, with neighborhood

these two as equivalent, it will be sufficient to talk about the neighborhood of $\omega$. Consider this arbitrary value for $f\left(z_{0}\right)=\omega_{0}$.

Where is it's neighborhood of values? Since $\omega$ comes from the evaluation of $f(z)$, we will examine some properties of $f(z)$ first. The function has a few properties that are advantageous. It's domain is all complex numbers, and it is a continuous function. It has a general left to right movement as you increase or decrease values of $z$ in the domain. In turn, the values of $\omega$ will rise and fall as small changes in $z$ occur. Thus, we will have a neighborhood of values around $\omega$ which are close by in the same manner that the values of $z$ are close-by each other. Even more so, there will be values of $\omega$ that are in the neighborhoods of each other that come from drastically different values of $z$ (think about a function that evaluates to the same point at different values of the domain; this implies that we may not have a one-to-one function).

How does having a neighborhood of $\omega$ actually help in the proof? Recall that we have picked $z_{0}$ such that it evaluates to the smallest $|\omega|, \mu$. What happens if $\mu$ is not on the origin of the complex plane? It will still have a neighborhood! In $\mu$ 's neighborhood will be other values of $|\omega|$ in all directions. This means with $\mu$ not on the origin, we will be able to find another $|\omega|$ smaller (or closer to the origin) than $\mu$. This will contradict our assumption that $\mu$ is the smallest value. This leaves $\mu$ to be at the origin. The neighborhood of the origin is all values within a circle that are not the origin, and therefore $\mu$ is the smallest $|\omega|$. Finally, since $\mu$ equals zero, it will follow that $\omega_{0}$ is zero giving every $f(z)$ a root.

So we now transform our empirical goal into a more structured mathematical one. Which part of our discussion of the neighborhoods should we exploit? The idea will be to take advantage of the contradiction by establishing that $\mu$ will not be the smallest value if it is greater than zero. So, as discussed earlier we assume $\mu>0$. Remember that $f\left(z_{0}\right)=\omega_{0}$ and $\left|f\left(z_{0}\right)\right|=\left|\omega_{0}\right|=\mu$.

Now consider the neighborhood of $z_{0}$, it is a circle; let's call it K. Inside of circle 


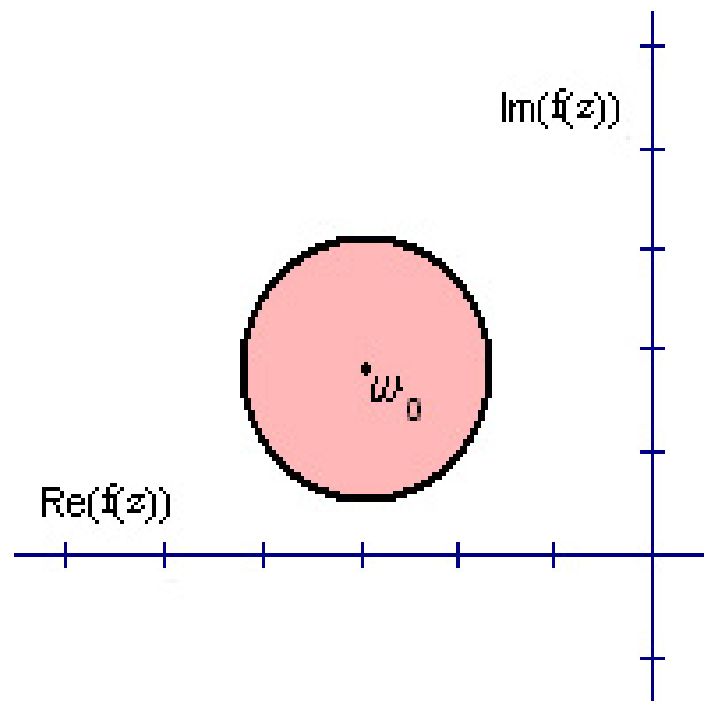

Figure 5: Visualization of $\omega_{0}$, with neighborhood

$\mathrm{K}$ are an infinite amount of values for $z$, but we do not want to consider these values independently. Instead, we want to define them in relation to $z_{0}$. Why is this intuitive? Since we show that $|\omega|$ is smaller than $\mu$ for one of these $z$ 's within K, if we define those $z$ 's in terms of $z_{0}$ it becomes much easier to compare values of $f(z)$ to $f\left(z_{0}\right)$ within K. So, we say that every $z$ within $K$ is a translation of $z_{0}$ such that $z=z_{0}+\zeta$, where $\zeta$ is a complex number. Now for every $z$ value we have a corresponding value for $\zeta$ from which it is constructed.

The immediate result of a new definition for $z$, is a new way to express $f(z)$.

$$
\begin{aligned}
f(z) & =z^{n}+C_{1} z^{n-1}+C_{2} z^{n-2}+\cdots+C_{n} \\
& =f\left(z_{0}+\zeta\right) \\
& =\left(z_{0}+\zeta\right)^{n}+C_{1}\left(z_{o}+\zeta\right)^{n-1}+C_{2}\left(z_{0}+\zeta\right)^{n-2}+\cdots+C_{n}
\end{aligned}
$$

By replacing $z$ with $z_{0}+\zeta$ we end up with a new way to express $f(z)$. The idea now would be to use this to our advantage. It may not be completely clear to see in the equation above, but the advantage is that the new expression, $f\left(z_{0}+\zeta\right)$, contains $f\left(z_{0}\right)$. This is a useful result because we know that $f\left(z_{0}\right)=\omega_{0}$, and remember our goal is to show that $\omega_{0}$ does not result in the smallest absolute magnitude. Thus having an expression with $\omega_{0}$ will be very useful, once we obtain $f\left(z_{0}\right)$ from $f\left(z_{0}+\zeta\right)$.

Returning to the new form of $f(z)=f\left(z_{0}+\zeta\right)$ reached, we have a sum of powers of $z_{0}$ and $\zeta$, and we now extract our original expression for $f\left(z_{0}\right)$. So, we are looking an extraction of all of the powers of $z_{0}$. We begin by examining arbitrary terms of the current derivation of $f\left(z_{0}+\zeta\right)$. Every term in the function is of the form $C_{k}\left(z_{0}+\zeta\right)^{k}$. Let's use the binomial theorem to expand a term of this form. 


$$
\begin{gathered}
(r+s)^{n}=\sum_{m=0}^{n}\left(\begin{array}{l}
n \\
m
\end{array}\right) r^{m} s^{n-m} \\
C_{k}\left(z_{0}+\zeta\right)^{k}=C_{k}\left(\begin{array}{c}
k \\
0
\end{array}\right) \zeta^{k}+C_{k}\left(\begin{array}{l}
k \\
1
\end{array}\right) z_{0} \zeta^{k-1}+C_{k}\left(\begin{array}{l}
k \\
2
\end{array}\right) z_{0}^{2} \zeta^{k-2}+\cdots+ \\
+C_{k}\left(\begin{array}{c}
k \\
k-2
\end{array}\right) z_{0}^{k-2} \zeta^{2}+C_{k}\left(\begin{array}{c}
k \\
k-1
\end{array}\right) z_{0}^{k-1} \zeta+C_{k}\left(\begin{array}{l}
k \\
k
\end{array}\right) z_{0}^{k}
\end{gathered}
$$

The above is the form of the expansion of every term in $f\left(z_{0}+\zeta\right)$. Note that there are $n+1$ such terms, where $n$ is the degree of the original polynomial; there are $n+1$ terms in $f(z)$, so $n+1$ terms in $f\left(z_{0}\right)$ and $f\left(z_{0}+\zeta\right)$. Now each individual term of the form $C_{k}\left(z_{o}+\zeta\right)^{k}$ has in itself $k+1$ terms, where each term is a distinct value of $k$ between zero and $n$. So now we can find the total number of terms in the complete expansion of $f\left(z_{0}+\zeta\right)$. The first term, $C_{n}\left(z_{0}+\zeta\right)^{n}$ will have $n+1$ terms, the second will have $n$ terms, the third $n-1$ terms, and so on until the last term, which has 1 term. Thus we need the sum $n+1$ down to 1 , or better, the sum from 1 to $n+1$. Thus from Gauss we wish to use the formula for the sum from 1 to $n$, in our case we take $n$ to be $n+1$.

$$
\begin{aligned}
\sum_{1}^{n} i & =\frac{n(n+1)}{2} \\
n=n+1 & \\
\sum_{1}^{n+1} i & =\frac{(n+1)(n+2)}{2}
\end{aligned}
$$

Thus, the entire expansion of $f\left(z_{0}+\zeta\right)$ has $\frac{(n+1)(n+2)}{2}$ terms. Now recall that we are extracting $f\left(z_{0}\right)$ from the complete expansion. Since $f\left(z_{0}\right)$ has only $n+1$ terms, there will be many terms left over after the extraction. Before worrying more about all the extra terms, let's first do the actual extraction of the terms that form $f(z)$. Since every $C_{k}\left(z_{0}+\zeta\right)^{k}$ ends with the term $C_{k}\left(\begin{array}{l}k \\ k\end{array}\right) z_{0}^{k}$ (see expansion above, and note $\left(\begin{array}{l}k \\ k\end{array}\right)=1$ ) we have a term of the form $C_{k} z_{0}^{k}$ for every $k$. Since $k$ runs from zero up to $n$ we will have a list of terms

$$
f\left(z_{0}\right)=z_{0}^{n}+C_{1} z_{0}^{n-1}+C_{2} z_{0}^{n-2}+\cdots+C_{n-1} z_{0}^{1}+C_{n}
$$

With a completely expanded $f\left(z_{0}+\zeta\right)$, we can collect terms such that the terms will fall into two groups; those terms which make up $f\left(z_{0}\right)$ and those which do not. Since 
we noted earlier that our proof will involve the use of $\omega_{0}$, let's denote all those terms which collect to be $f\left(z_{0}\right)$ as $\omega_{0}$. For the terms that collect to not be in $f\left(z_{0}\right)$, let's denote them as $\Gamma$. Now we can rewrite $f\left(z_{0}+\zeta\right)$ after expanding and collecting the terms. Also remember that $f\left(z_{0}+\zeta\right)$ was equivalent to any $f(z)$ within our circle, $\mathrm{K}$.

$$
f(z)=f\left(z_{0}+\zeta\right)=\omega_{0}+\Gamma
$$

We now have $\omega_{0}$ within our expression for $f\left(z_{0}+\zeta\right)$. To show that $\omega_{0}$ does not produce the smallest $|\omega|$, we need a direct way to show $\left|\omega_{0}\right|>|\omega|$ for some arbitrary $\omega$ in K. Although this might immediately seem like a large obstacle to overcome, it is a problem remedied by one of our first assignments, $f(z)=\omega$. With this equality we restate $f\left(z_{0}+\zeta\right)$ again, leaving an equation with two values of the absolute magnitude, $\omega_{0}$ and $\omega$, another step toward showing that $\left|\omega_{0}\right|=\mu$ is not the smallest $|\omega|$.

$$
\begin{gathered}
\omega=f(z)=f\left(z_{0}+\zeta\right)=\omega_{0}+\Gamma \\
\omega=\omega_{0}+\Gamma
\end{gathered}
$$

The next step will be to examine $\Gamma$ so that we establish a relationship between $\omega$ and $\omega_{0}$. Since $f\left(z_{0}+\zeta\right)=f\left(z_{0}\right)+\Gamma$, the question is how to deal with all those extra terms that we forced into $\Gamma$. To accomplish this, we first aim to achieve some ordering to the terms in $\Gamma$, and so we begin by organizing the terms in increasing powers of $\zeta$. There are various complex constants and powers of $z_{0}$ attached to each such $\zeta^{k}$. For instance, when we expanded the terms $C_{k}\left(z_{0}+\zeta\right)^{k}$, there was a $\zeta^{2}$ term produced for every binomial term with $k \geq 2$. Once we group all terms by their respective powers of $\zeta$, the immediate step is to factor out that power of $\zeta$ from the group of terms. For example

$$
\zeta^{k}\left(C_{1}+C_{2} z_{0}^{k}-2+C_{9} z_{0}^{k}-9\right)
$$

Let's use this term to continue our examination of $\Gamma$. It is good to note that we cannot do much at the moment with $\zeta$, as it is a variable representing all translations of $z_{0}$ within K. But the sum it is multiplied by, is it that difficult to manipulate? First, the powers of $z_{0}$ are easy to handle because $z_{0}$ is a particular value of $z$. $z_{0}$ is multiplied by various constants, also particular as they are given in the original statement of $f(z)$. So, the sum is merely a complex number. Let's refer to those complex numbers as $c$. Now we have each term in $\Gamma$ as a product of powers of $\zeta$ and $c$, a complex number. Stepping back to our statement of $f\left(z_{0}+\zeta\right)$ we have

$$
\begin{aligned}
& f\left(z_{0}+\zeta\right)=\omega_{0}+\Gamma \\
& f\left(z_{0}+\zeta\right)=\omega_{0}+c_{1} \zeta+c_{2} \zeta^{2}+\cdots+c_{n} \zeta^{n}
\end{aligned}
$$

Now we have a more comfortable expression of $f\left(z_{0}+\zeta\right)$ with which to work. Note 
that the new constant terms, $c_{i}$, are dependant on $z_{0}$. Since we aim for a relationship between $\omega$ and $\omega_{0}$, we see that through division by $\omega_{0}$ there is a ratio of $\omega$ to $\omega_{0}$. However, does this cause a problem with the terms from $\Gamma$ ? Just as we were able to manipulate $\Gamma$ with our particular value for $z_{0}$, we now divide by $\omega_{0}$, a particular value for $\omega$. Thus the division can be accomplished, and all terms $\frac{c_{i}}{\omega_{0}}$ are denoted $q_{i}$, and remember these are still constants dependant on $z_{0}$.

$$
\begin{gathered}
\omega=f(z)=f\left(z_{0}+\zeta\right)=\omega_{0}+c_{1} \zeta+c_{2} \zeta^{2}+\cdots+c_{n} \zeta^{n} \\
\omega=\omega_{0}+c_{1} \zeta+c_{2} \zeta^{2}+\cdots+c_{n} \zeta^{n} \\
\frac{\omega}{\omega_{0}}=1+q_{1} \zeta+q_{2} \zeta^{2}+\cdots+q_{n} \zeta^{n}
\end{gathered}
$$

With a ratio established, we now manipulate the right side of our equation exclusively. What do we want the right side to tell us? Since the left side is a ratio with $\omega_{0}$ as the denominator, if the right side of the equation is less than 1 , then $\omega$ will have to be smaller than $\omega_{0}$. Then it will only remain to show that this implies that the same relationship occurs for the absolute magnitudes of $\omega$ and $\omega_{0}$. How is it that we can make the right side of the equation less than 1? Notice the first term in the right side, it is 1 . Thus if all the other terms are shown to be negative, the right side will be some number less than 1. At this point we denote all the terms which are not 1 in the right side of the equation as $\Phi$

$$
\begin{gathered}
\frac{\omega}{\omega_{0}}=1+\Phi \\
\Phi=q_{1} \zeta+q_{2} \zeta^{2}+\cdots+q_{n} \zeta^{n}
\end{gathered}
$$

It does not seem viable to show that every individual term of $\Phi$ is a negative number. It would be much more useful for the terms to simply be a multiple of a negative number. Before worrying about the negative aspect of the multiple, we need $\Phi$ to have a common multiple. Since they are all complex numbers and powers of $\zeta$ is is sufficient to again do some factorization. We extract the first term of $\Phi$ from every term in $\Phi$, and this is always possible because we had already ordered $\Phi$ by increasing powers of $\zeta$. Once, $q_{1}$ has been factored out of all the other $q_{i}$ 's, denote the constants as $p_{i}$ 's, and simply refer to $q_{1}$ as q. Again, remember that these constants are dependant on $z_{0}$.

$$
\Phi=q \zeta\left(1+p_{1} \zeta+p_{2} \zeta^{2}+\cdots+p_{n-1} \zeta^{n-1}\right)
$$

Now the term $q \zeta$ is essentially a multiple of $\Phi$. So the goal is to show that $q \zeta$ is negative. What about the remaining part of $\Phi$ ? It is 1 plus powers of $\zeta$, but it is not important to think of this as such a long, expansive sum. So we denote the powers of $\zeta$ with their respective $p_{i}$ values as $\xi$. For now, this will seem like more of a notational 
trick with which to shorten what we write, but after obtaining $q \zeta$ as negative, we will return to $(1+\xi)$. We now have a clean way of observing our ratio

$$
\frac{\omega}{\omega_{0}}=1+q \zeta(1+\xi)
$$

To show that $q \zeta$ is negative, we begin with the reminder that each $q$ and $\zeta$ are complex numbers. We will find use in considering them in their polar form.

$$
\begin{aligned}
& \zeta=\rho(\cos \theta+i \sin \theta) \\
& q=h(\cos \lambda+i \sin \lambda)
\end{aligned}
$$

Now with the product of $q$ and $\zeta$ we get a useful result, as multiplying complex numbers simply sums their angles and multiplies their magnitudes.

$$
q \zeta=\rho h(\cos (\theta+\lambda)+i \sin (\theta+\lambda))
$$

How does this form of $q \zeta$ help force the value to be negative? Consider that since the product $q \zeta$ is a complex number, that it has been rewritten as a product of it's absolute magnitude, $\rho h$, and it's angular component, $\cos (\theta+\lambda)+i \sin (\theta+\lambda)$. Remember that since $\theta$ depends on $\zeta$ it will be an arbitrary value and can thus be any angle we choose. Also note that $\lambda$ will not be arbitrary as it depends on $z_{0}$.

Beginning with the first term in the restatement of $q \zeta$, we see that $\rho h$ is always positive. As discussed earlier, absolute magnitudes describe a distance, and they are therefore always positive. That leaves $\cos (\theta+\lambda)+i \sin (\theta+\lambda)$ to be made negative. Observe that this component of $q \zeta$ is a complex number, and this is not what is wanted. A definite negative is needed; how can this be accomplished? Elimination of the complex part could result in a number that is always negative. To eliminate the complex part, $\sin (\theta+\lambda)$ must be zero. We must find an appropriate value of $\theta$ to get this result. This happens in two cases, when $\theta=2 \pi-\lambda$ or when $\theta=\pi-\lambda$. Each case seems equally useful, but remember once we fix $\theta$ it is also fixed for the real part of $\cos (\theta+\lambda)+i \sin (\theta+\lambda)$. The case of $\theta+\lambda=\pi$ falls out as the case to exploit. So let $\theta+\lambda=\pi$.

$$
\begin{aligned}
q \zeta & =\rho h(\cos (\theta+\lambda)+i \sin (\theta+\lambda)) \\
& =\rho h(\cos \pi+i \sin \pi) \\
& =\rho h(-1+i \cdot 0) \\
& =\rho h(-1) \\
& =-\rho h
\end{aligned}
$$

Thus we have $q \zeta$ as a negative number. Before continuing, let's ensure our selection of $\theta=\pi-\lambda$. The thing to clarify is the fact that we have the sufficient value of $\theta$ to 


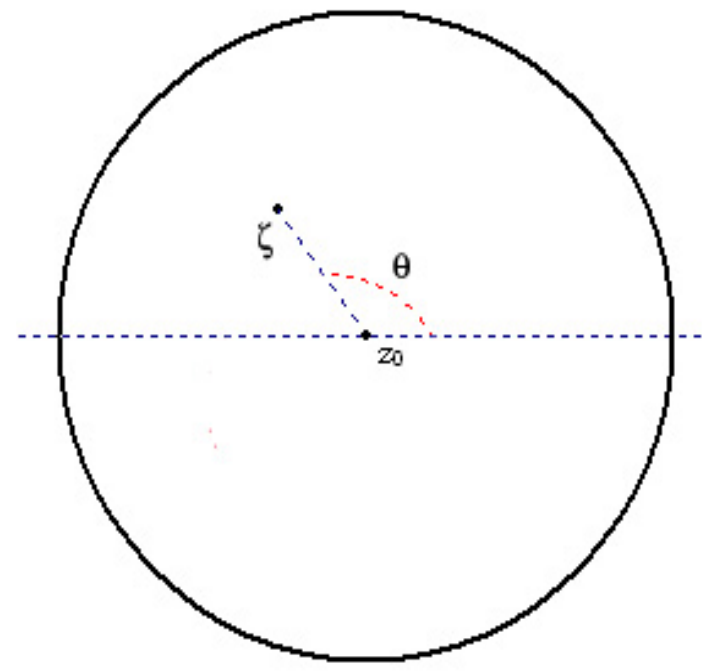

Figure $6: z_{0}, \zeta$, and $\theta$, the angle created by them

ensure it can always be $\pi-\lambda$. Remember $\theta$ is the angle that $\zeta$ makes relative to $z_{0}$, and this can be anywhere, for our work we consider it between $-2 \pi$ and $2 \pi$ depending on whether the angle is measured in a clockwise or counterclockwise manner.

Still, the idea is to choose $\theta$ so that it will be $\pi-\lambda$. Recall where $\zeta$ is from; $\zeta$ was an arbitrary value inside of $\mathrm{K}$. Remember that $q$ is the ratio between a constant of $z_{0}$ and $\omega_{0}$; it follows then that $\lambda$ is dependent on $\zeta$ alone. Now we pick a $\zeta$ in $\mathrm{K}$ such that our desired property $\theta=\pi-\lambda$ is met. Since $\mathrm{K}$ is a circle, this is always possible.

Having verified $q \zeta=-\rho h$ we have

$$
\frac{\omega}{\omega_{0}}=1-\rho h(1+\xi)
$$

We now re-examine $\xi$ in order to show it can be brought to a arbitrarily small value.

$$
\xi=p_{1} \zeta+p_{2} \zeta^{2}+\cdots+p_{n-1} \zeta^{n-1}
$$

We see that $\xi$ is a sum of products of complex constants $\left(p_{i}\right)$ and powers of $\zeta$. Familiar to earlier steps in the proof, $\zeta$ is factored from each term.

$$
\xi=\zeta\left(p_{1}+p_{2} \zeta+p_{3} \zeta^{2} \cdots+p_{n-1} \zeta^{n-2}\right)
$$

Now $\xi$ is an expression that contains a polynomial of $\zeta$.

$$
\xi=\zeta \cdot \mathrm{P}(\zeta)
$$

Why did we want to have this polynomial? We can now use the continuity of $\mathrm{P}(\zeta)$ together with $\mathrm{K}$ and say that it is now bounded from above and below and we write 
$|\mathrm{P}(\zeta)|<\mathrm{M}$. Since the circle is compact and $\mathrm{P}(\zeta)$ is continuous we use the fact that continuous functions on compact sets attains maximum and minimum values and is therefore bounded. Thus we see that by having the ability to make $\zeta$ arbitrarily small we likewise make a polynomial of $\zeta$ arbitrarily small. Now with both quantities that compose $\xi$ being made arbitrary small by virtue of $\zeta$, we make $|\xi|$ small as well.

$$
\begin{aligned}
|\xi| & =|\zeta \cdot \mathrm{P}(\zeta)| \\
& =|\zeta| \cdot|\mathrm{P}(\zeta)| \\
& <|\zeta| \cdot \mathrm{M}
\end{aligned}
$$

Although it may have been the intuition to try and make $\xi$ both a real number and arbitrarily small, we now recall the fact that we are actually looking to say something about absolute magnitude of the ratio $\frac{\omega}{\omega_{0}}$. When examining the absolute magnitude, the fact that $\xi$ is not a complex number is excusable. So it only remains to show, for some $z$ in $\mathrm{K}$ that

$$
\left|\frac{\omega}{\omega_{0}}\right|<1
$$

This is accomplished by taking the absolute magnitude of our current expression for $\frac{\omega}{\omega_{0}}$ and then applying the knowledge gained about the ratio thus far.

$$
\begin{aligned}
\frac{\omega}{\omega_{0}} & =1-\rho h(1+\xi) \\
\left|\frac{\omega}{\omega_{0}}\right| & =|1-\rho h(1+\xi)| \\
& =|1-\rho h-\rho h \cdot \xi| \\
& \leq|1-\rho h|+|\rho h| \cdot|\xi| \\
& =1-\rho h+\rho h|\xi| \\
& =1+\rho h(|\xi|-1)
\end{aligned}
$$

Following above, we first take the absolute magnitude of the ratio (1). After algebraic distribution, we use the triangle inequality to get (2). Now returning to $\rho h$ we remember that this quantity is dependent on $\zeta$, for $\rho$ is the absolute magnitude of $\zeta$, and thus as we make $\rho h$ small. Also recall this quantity to be positive, thus in (3) we can drop each absolute value associated with $\rho h$. A factorization of (3) brings us to (4) and we now write

$$
\left|\frac{\omega}{\omega_{0}}\right| \leq 1+\rho h(|\xi|-1)
$$

Now we recall that we are able to make $|\xi|$ arbitrarily small. It follows that $|\xi|-1<0$. With this quantity negative multiplied by $\rho h$, which can also be made arbitrarily small we finally have 


$$
\left|\frac{\omega}{\omega_{0}}\right|<1
$$

We now have $\left|\frac{\omega}{\omega_{0}}\right|<1$ whenever $\frac{\omega}{\omega_{0}}<1$. This result gives us the inequality $|\omega|<\left|\omega_{0}\right|$, and this is a contradiction. Recall that $\left|\omega_{0}\right|=\mu$, where $\mu$ was the smallest of all absolute magnitudes. We have established that there is a $z$ in the neighborhood $\mathrm{K}$ that has an absolute magnitude less than the absolute magnitude designated as the smallest. This contradiction counteracts our assumption that $\mu>0$, and therefore $\mu=0$.

Finally, we have $\mu=\left|\omega_{0}\right|=\left|f\left(z_{o}\right)\right|=0$. The only situation in which $\left|f\left(z_{0}\right)\right|=$ $\left|\omega_{0}\right|=0$ is when $f\left(z_{0}\right)=0$. Consider a value for $f\left(z_{0}\right) \neq 0$; this value has to be at some non-origin point on the complex plane, thereby giving the function and absolute distance greater than zero. We cannot have an absolute magnitude greater than zero. So, every complex polynomial equation has at least one root.

Fundamental Theorem of Algebra 2 Any equation of the form

$$
z^{n}+C_{1} z^{n-1}+C_{2} z^{n-2}+\cdots+C_{n}=0
$$

has exactly $n$ roots.

With the result obtained from the first part of the theorem, we now show that every complex polynomial has exactly $n$ roots. To obtain this result we must first obtain the following corollary.

Fundamental Theorem Corollary 1 Any equation $f(z)=0$ with a root $\alpha$ can be divided evenly by the factor $z-\alpha$.

We begin with $f(z)$ of degree $n$, dividing it by $z-\alpha$. Following what we know about division and division of polynomial equations, we have that this division will result in a new polynomial with degree $n-1$ and a remainder, $R$. The remainder is a constant term not evenly divisible by $z-\alpha$.

$$
\frac{f(z)}{z-\alpha}=f_{1}(z)+\frac{R}{z-\alpha}
$$

The corollary speaks directly to the nature of $f(z)$, thus we solve the equation for $f(z)$ with multiplication by $z-\alpha$.

$$
f(z)=(z-\alpha) f_{1}(z)+R
$$

To show that $f(z)$ is evenly divisible, it remains to eliminate the remainder. Consider that $f(z)$ is valid for all $z$. By the instance of $z=\alpha$ we have $R=0$. Also remember that since $\alpha$ is a root of $f(z), f(\alpha)=0$. 


$$
\begin{aligned}
f(\alpha) & =(\alpha-\alpha) f_{1}(\alpha)+R \\
0 & =0+R \\
0 & =R
\end{aligned}
$$

We have $R=0$ for one choice of $z$. This will allow the remainder to be zero for all choices of $z$. Remember, $f(z)$ has the same root $\alpha$ for all choices of $z$. That implies that if there is a restriction on $R$ for any particular $z$, it must also hold for all other choices of $z$. Therefore we can evenly divide $f(z)$ using it's root.

$$
f(z)=(z-\alpha) f_{1}(z)
$$

Using this corollary we can complete the theorem. We now have any complex polynomial as a product of one of it's roots and a polynomial of one less degree. This polynomial is also a product of one of it's roots and a polynomial of one less degree, and so on.

$$
\begin{aligned}
f(z) & =\left(z-\alpha_{1}\right) f_{1}(z) \\
f_{1}(z) & =\left(z-\alpha_{2}\right) f_{2}(z) \\
f_{2}(2) & =\left(z-\alpha_{3}\right) f_{3}(z)
\end{aligned}
$$

We observe that these factorizations will continue, but until what point? In our factorizations, it is necessary that the polynomial have another polynomial with one less degree as one of it's factors. So, once the polynomial is factorized $(n-1)$ times, it will be a product of $(n-1)$ roots along with a polynomial of degree one. But a polynomial of degree one is a linear function. Notice that our roots are linear functions of $z$. So the $(n-1)$ th factorization of $f(z)$ is the final factorization needed, as the remaining polynomial is itself a root of $f(z)$.

$$
\begin{aligned}
f(z) & =\left(z-\alpha_{1}\right) f_{1}(z) \\
f(z) & =\left(z-\alpha_{1}\right)\left(z-\alpha_{2}\right) f_{2}(z) \\
\vdots & \\
f(z) & =\left(z-\alpha_{1}\right)\left(z-\alpha_{2}\right) \cdots\left(z-\alpha_{n-1}\right) f_{n}(z) \\
f(z) & =\left(z-\alpha_{1}\right)\left(z-\alpha_{2}\right) \cdots\left(z-\alpha_{n-1}\right)\left(z-\alpha_{n}\right)
\end{aligned}
$$

Lastly, we show that there are no more than these $n$ roots. Recall that we have $f(z)=0$.

$$
f(z)=\left(z-\alpha_{1}\right)\left(z-\alpha_{2}\right) \cdots\left(z-\alpha_{n}\right)=0
$$


Here we see that it is only necessary for one of the factors of $f(z)$ to be zero and the entire product will evaluate to zero. We write $z-\alpha_{i}=0$ and we have $z=\alpha_{i}$ for every $i=1,2, \ldots, n$. And since we have written $f(z)$ as a product of all it's roots, $f(z)$ has $n$ roots exactly, and the proof is complete. 


\section{Conclusion}

Having proven the Fundamental Theorem of Algebra, we return to a topic at the outset of the discussion, that being how the theorem is applied in a high school environment. The main obstacle with presenting the theorem to high school students is that you cannot present it in it's complete form. The idea of a complex polynomial is totally foreign to the high school curriculum; it is not a complete stretch to say that an average high school student may think complex numbers only exist as answers to problems rather than the beginning of problems themselves.

The watered down version of the theorem that tends to appear in high school texts usually says something to the effect of any polynomial equation of degree $n$ will have $n$ roots, where those roots can be real, complex, or both, and they are not necessarily unique. As discussed in the opening of the paper, this theorem will not impress upon students it's importance, as they are already used to finding two solutions to quadratic equations for instance.

A successful way to communicate the power of the theorem to students may then be to present problems in an abstract sense. This may be in part analogous to teaching students the derivation of the quadratic formula, where after learning to factor, complete the square, etc, they are able to take a quadratic equation and find that it will always have two solutions.

We close this discussion of the fundamental theorem with a few problems regarding the theorem in an abstract sense that a high school student may encounter. The problems have been taken from the third edition of "Precalculus with Limits: A Graphing Approach." 
Exercise 1. Graph the function $f(x)=x^{4}-4 x^{2}+k$ for different values of $k$. Find when $k$ has four real zeros, two real zeros and two complex zeros, and four complex zeros.

Assuming that the student begins with integer values for $k$, he or she will soon find that the two values of interest are zero and 4 . It will soon follow that 4 zeros occur when $0<k<4$, two of each occur when $k<0$, and four complex zeros occur when $k>4$. This is accomplished first by the knowledge the theorem gives that $f(x)$ will have 4 roots by the fact that it is of the 4 th degree. Then by the knowledge that 4 real zeros will have graphs that cross the x-axis in 4 places, 2 of each when the graph crosses in 2 places, and 4 complex when the graph never crosses. Here are examples of the three graphs a student may attain with $k=2, k=-2$, and $k=6$ for 4 , 2, and zero real roots respectively.
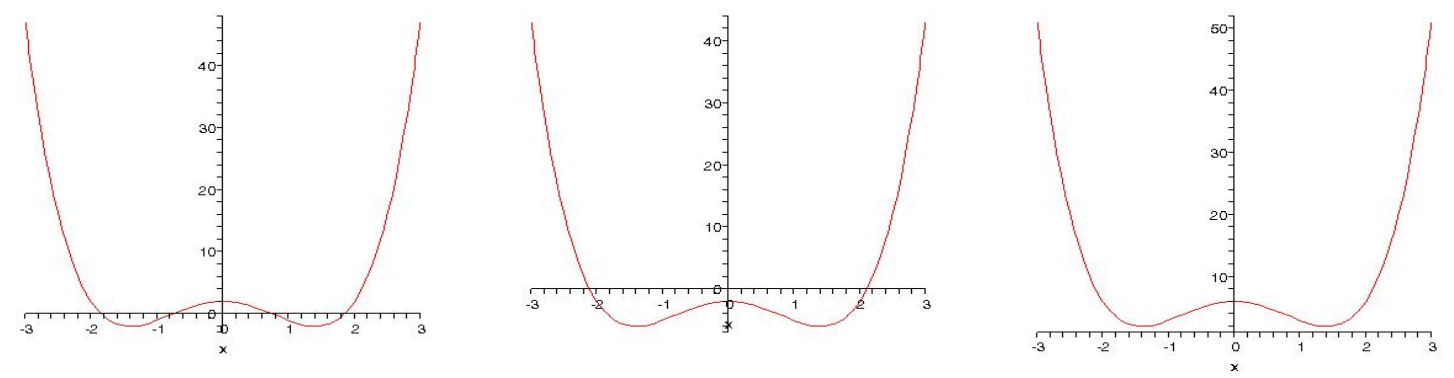

Although a more difficult task, student can also arrive at these solutions without the aid of graphs through knowledge of the quadratic formula. Since $f(x)$ has the form $a x^{2}+b x+c$ where $x=x^{2}$ the quadratic formula is used to find solutions for $x^{2}$, and taking those solutions square root, four solutions for $x$ are found.

$$
\begin{aligned}
x^{2} & =\frac{4 \pm \sqrt{16-4 k}}{2} \\
x & = \pm \sqrt{\frac{4 \pm \sqrt{16-4 k}}{2}}
\end{aligned}
$$

We observe that $x$ will produce 4 solutions by virtue of the two radical terms. The outer radical term determines the nature of the roots. Beginning with the simplest case, 4 complex roots, the expression underneath the inner radical will be sufficient to make all the solutions for $x$ complex.

$$
\begin{aligned}
\sqrt{16-4 k} & <0 \\
16-4 k & <0 \\
16 & <4 k \\
4 & <k
\end{aligned}
$$


Thus when $k>4$ there are 4 complex roots. Next, for $x$ to have 4 real solutions, observe that each radical expression must be positive.

$$
\begin{aligned}
\sqrt{16-4 k} & >0 \\
16-4 k & >0 \\
16 & >4 k \\
4 & >k \\
4 \pm \sqrt{16-4 k} & >0 \\
4 & >\sqrt{16-4 k} \\
16 & >16-4 k \\
0 & >-4 k \\
0 & <k
\end{aligned}
$$

So we have 4 real roots when $0<k<4$. Finally, the case of 2 complex roots and 2 real roots. This will occur only when the \pm component of the outer radical splits the solutions into two groups. Thus we will have 2 complex, 2 real when $4+\sqrt{16-4 k}>0$ and $4-\sqrt{16-4 k}<0$. These two expressions are actually the same, we show this by algebraic manipulation of the first inequality.

$$
\begin{aligned}
4+\sqrt{16-4 k} & >0 \\
4 & >-\sqrt{16-4 k} \\
-4 & <\sqrt{16-4 k} \\
16 & <16-4 k \\
4 & <\sqrt{16-4 k} \\
4-\sqrt{16-4 k} & <0
\end{aligned}
$$

Solving either inequality will be sufficient, so continuing from (5)

$$
\begin{aligned}
16 & <16-4 k \\
0 & <-4 k \\
0 & >k
\end{aligned}
$$

Thus, 2 roots each of complex and real results when $k<0$. So we have a second way to find a solution given our knowledge thanks to the fundamental theorem that $f(x)$ will always have 4 roots combined with knowledge of the quadratic equation and inequalities. 
Exercise 2. Find the appropriate polynomial function associated with the roots $a \pm b i$. The function has these roots and no more.

First we observe that the solution will be a quadratic function, because by the fundamental theorem an equation with exactly 2 roots will be a 2 nd degree polynomial. To find the solution we also use the corollary to the fundamental theorem that a function can be evenly factored by it's roots. Thus we know the polynomial will be the product of $(x-(a+b i))$ and $(x-(a-b i))$.

$$
\begin{aligned}
f(x) & =(x-(a+b i))(x-(a-b i)) \\
& =x^{2}-x(a+b i)-x(a-b i)+(a+b i)(a-b i) \\
& =x^{2}-x(a+b i+a-b i)+\left(a^{2}+a b i-a b i-b^{2} i^{2}\right) \\
& =x^{2}-2 a x-\left(a^{2}+b^{2}\right)
\end{aligned}
$$

So the polynomial function formed by the roots $a \pm b i$ is $f(x)=x^{2}-2 a x-\left(a^{2}+b^{2}\right)$. 


\section{References}

(1)Cajori, Florian. (1985). A History of Mathematics (4th ed.). New York: Chelsea.

(2)Dörrie, H. (1965). 100 Great Problems of Elementary Mathematics: Their History and Solution. In D. Antin (Trans.), Gauss' Fundamental Theorem of Algebra (Ch. 23 pp. 108-112). New York: Dover. (Original work published in 1958)

(3)Larson, R., Hostetler, R. P., \& Edwards, B. H. Precalculus with Limits A Graphing Approach. In J. Shira (Ed.), The Fundamental Theorem of Algebra (pp. 182-188). Boston: Houghton Mifflin.

(4)O'Connor, J. J., \& Robertson E. F. (March 2005). The Fundamental Theorem of Algebra, The Fundamental Theorem of Arithmetic, \& Johann Carl Friedrich Gauss.

Retrieved December 2005, from http://www-groups.dcs.st-and.ac.uk/ history/index.html

(5)Wikipedia. (Current). Various articles on mathematics and the history of mathematics including but not limited to Fundamental Theorem of Algebra, Carl Friedrich Gauss, and Complex Numbers. Retrieved Nov-Dec 2005, from http://en.wikipedia.org 